\title{
Corela
}

Cognition, représentation, langage

HS-22 | 2017

Prédication et prépositions en anglais

\section{Le traitement des prépositions en grammaire générative}

Jean-Charles Khalifa

\section{(2) OpenEdition}

Journals

Édition électronique

URL : http://journals.openedition.org/corela/4949

DOI : $10.4000 /$ corela.4949

ISSN : 1638-573X

Éditeur

Cercle linguistique du Centre et de l'Ouest - CerLICO

Référence électronique

Jean-Charles Khalifa, «Le traitement des prépositions en grammaire générative », Corela [En ligne], HS-22 | 2017, mis en ligne le 19 juin 2017, consulté le 01 mai 2019. URL : http://

journals.openedition.org/corela/4949; DOI : 10.4000/corela.4949

Ce document a été généré automatiquement le 1 mai 2019.

\section{(c) (i) (2)(2)}

Corela - cognition, représentation, langage est mis à disposition selon les termes de la licence Creative Commons Attribution - Pas d'Utilisation Commerciale - Partage dans les Mêmes Conditions 4.0 International. 


\title{
Le traitement des prépositions en grammaire générative
}

\author{
Jean-Charles Khalifa
}

\section{Introduction : la préposition en tant que partie du discours}

1 Il est tout d'abord intéressant de noter que, dans les traitements classiques remontant à Chomsky 1965 et 1970, la préposition est présentée comme une des quatre grandes parties du discours, autrement dit des quatre catégories lexicales, lesquelles sont définies en termes de combinaisons de deux traits syntaxiques distinctifs, $\{ \pm \mathrm{N}\}$ (nominal ou nonnominal) et $\{ \pm V\}$ (verbal ou non-verbal). C'est la présence ou l'absence de ces deux traits qui nous donne la matrice suivante (Figure 1) :

\begin{tabular}{|l|l|l|}
\hline \multicolumn{2}{|l|}{$\mathrm{V}$} & \multicolumn{2}{l|}{$\mathrm{N}$} \\
\hline Nom (N) & - & + \\
\hline Verbe (V) & + & - \\
\hline Adjectif (A) & + & + \\
\hline $\begin{array}{l}\text { Préposition } \\
\text { (P) }\end{array}$ & - & - \\
\hline
\end{tabular}

Figure 1

2 qui se lit ainsi :

- la catégorie du Nom (N) se définit comme exclusivement nominale et exclusivement non-verbale : un nom n'a que des traits nominaux, et aucun trait verbal. 
- la catégorie du Verbe (V) présente les traits exactement opposés: un verbe est exclusivement verbal et n'a aucun des traits du nom. Il y a donc opposition structurale entre les deux catégories $\mathrm{N}$ et $\mathrm{V}$.

- la catégorie de l'Adjectif (A) présente en revanche à la fois des traits nominaux (souvenons-nous que les grammairiens classiques, et en tout premier la Grammaire de Port-Royal (Arnauld et Lancelot, 1660) distinguaient les «noms substantifs» des «noms adjectifs »), et verbaux (l'adjectif a des fonctions prédicatives qui le rapprochent beaucoup du verbe).

3 Reste donc notre quatrième catégorie, $\mathrm{P}$, qui quant à elle, apparemment, ne se définit que négativement, comme n'ayant aucun des traits du verbe et aucun des traits du nom. Mais, lorsque l'on y regarde de plus près, Chomsky en réalité ne faisait pas, dans les écrits cités, entrer la préposition dans le système. La quatrième ligne du tableau n'existait pas et ce n'est que quelques années plus tard que Jackendoff (1977) complète le tableau en y inscrivant la catégorie: 'A fourth major syntactic category is $\mathrm{P}$ (reposition), about which we will have much to say in this study. Presumably Chomsky's system would analyze it as $[-\mathrm{N},-\mathrm{V}]$, completing the matrix in 3.3' (p. 31). Les motivations pour introduire la préposition en tant que grande catégorie lexicale à égalité de statut avec $\mathrm{N}, \mathrm{V}$ ou A semblent donc relever pour une bonne part d'une préoccupation plus structurale que purement linguistique (et d'ailleurs l'inconscient de Jackendoff semble aussi le trahir lorsqu'il écrit 'syntactic category' et non lexical...). Il n'empêche que cette théorie des 4 grandes parties du discours est très largement acceptée depuis en linguistique, l'une des exceptions les plus notables étant celle, en 2003 de Baker ${ }^{1}$, qui montre dès le titre de son ouvrage (Lexical Categories: Verbs, Nouns, and Adjectives) qu'il conteste l'appartenance des prépositions aux grandes catégories lexicales:

But this theory is widely recognized to have almost no content in practice. The feature system is not well integrated into the framework as a whole, in that there are few or no principles that refer to these features or their values. (p. 14)

4 Tel qu'il est présenté, le système permet en principe d'opérer des rapprochements et d'analyser les catégories en termes de leurs traits communs et de leurs traits distinctifs. On peut par exemple remarquer que les catégories $\mathrm{N}$ et $\mathrm{V}$ n'ont aucun trait commun, et s'opposent donc très exactement l'une à l'autre. En revanche, on a déjà observé que la catégorie A présentait un trait commun à la fois avec $\mathrm{N}(\{+\mathrm{N}\})$ et avec $\mathrm{V}(\{+\mathrm{V}\})$. Quant à la catégorie $\mathrm{P}$, elle présente un trait commun avec $\mathrm{V}$, à savoir le trait $\{-\mathrm{N}\}$, et un trait commun avec $\mathrm{N}$, à savoir $\{-\mathrm{V}\}$. Les catégories présentant des traits communs sont réputées former des supercategories dotées de propriétés communes. C'est ainsi que l'on peut trouver une explication unitaire à un certain nombre de faits en apparence disparates, dont nous ne donnons pas le détail $\mathrm{ici}^{2}$, mais nous attarderons sur celle-ci, qui sera fondamentale pour notre propos: le cas accusatif apparait uniquement dans les contextes suivants :

[1a] Mary loves him / *he (cf. I suppose you want someone to identify her (l. 15))

[1b] Mary is very fond of him / *he (cf. Nothing was left of her (1.4))

5 c'est-à-dire que les catégories ayant la propriété d'assignation du cas objet à leur complément sont $\mathrm{V}$ et $\mathrm{P}$, donc celles qui partagent le trait $\{-\mathrm{N}\}$. Nous y revenons cidessous en détail, mais remarquons tout de suite, avec Baker, que les contre-exemples à cette idée de supercategory sont relativement faciles à trouver: par exemple si l'on regarde les énoncés [2], on s'aperçoit que, dans ces constructions résultatives, ce sont les 
adjectifs et les prépositions, lesquels a priori ne partagent aucun trait, qui semblent former une classe :

[2a] John pounded the metal flat (AP)

[2b] John threw the ball into the barrel (PP)

[2c] *John pounded the metal a sword (NP)

[2d] *John polished the table shine (VP)

On pourrait dire aussi, dans le même sens, que adjectifs et prépositions sont susceptibles de modifier des noms (the man responsible / the man in the garden) et que seules ces deux catégories peuvent être modifiées par des adverbiaux de mesure (It is three yards long / He went three yards into the water, ou encore mieux, dans notre texte: The car fell a hundred feet into the ravine (1.2)). En résumé, même si le système de traits binaires est encore largement accepté et exploité, en particulier dans les manuels de syntaxe (par exemple, Radford 1997, mais on notera qu'il est absent dans Radford 2009), il n'a qu'une valeur relative.

En fait, tout le débat qui se profile derrière cette controverse, qui peut sembler, au fond, de peu de conséquence pour l'analyse syntaxique, est celui de la nature même de cette catégorie. Si elle n'est pas lexicale, alors elle est forcément fonctionnelle, selon une dichotomie qui remonte aux grands grammairiens descriptivistes ${ }^{3}$ et sur laquelle nous reviendrons. Nous passerons d'abord par quelques ${ }^{4}$ grands rappels théoriques sur le cadre générativiste.

\section{Rappel : structure du syntagme en syntaxe X-barre}

Que l'on accepte ou non l'analyse en supercategories et en matrices de traits binaires distinctifs, la préposition reste analysée comme une tête (head), laquelle comme toute tête dans la théorie se projette en un syntagme (phrase), par fusion (merge) tout d'abord avec un complément (sœur de la tête), puis un spécifieur (sœur de la projection intermédiaire ainsi obtenue) : toute relation, dans ces théories, est réputée binaire, un

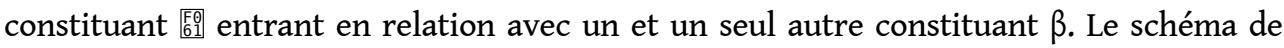
base, universel, de tout syntagme, est donné infra en Figure 2 et illustré en Figure 3. On notera que la position de complément comme celle de spécifieur peuvent être vides, ce qui peut donner des syntagmes comme en Figure 2' (spécifieur, mais pas de complément) ou Figure 2" (ni spécifieur, ni complément). Bien sûr, on utilisera avec profit le modèle pour distinguer les vraies prépositions, qui auront forcément un complément, des prépositions sans complément que l'on nommera différemment (particules, adverbes, ou autres, voir infra). La Figure 3 illustre un syntagme prépositionnel tiré du texte proposé (l. 2) où toutes les positions sont instanciées. On constate que la classe des constituants susceptibles d'instancier une position de spécifieur dans un PP est assez restreinte, en l'occurrence à des adverbes de degré et d'intensité (right, straight, exactly etc.). Quant à son complément, c'est nécessairement ${ }^{5}$ un groupe nominal : on sait que la préposition est un test, permettant entre autres de déterminer le degré de nominalité d'un constituant qui n'est pas catégoriellement nominal, par exemple une proposition. Ce test est utilisé par Jean-Marie Merle dans sa contribution (ce volume) dans les exemples (4) et (9) tirés du texte :

4. (1. 17-18) [...] furious with Laura for what she'd done, [...]

4'. [...] furious with Laura for some reason, [...]

9. (l. 1) Ten days after the war ended, my sister Laura drove a car off a bridge.

9 ' Ten days after the end of the war, my sister Laura drove a car off a bridge. 


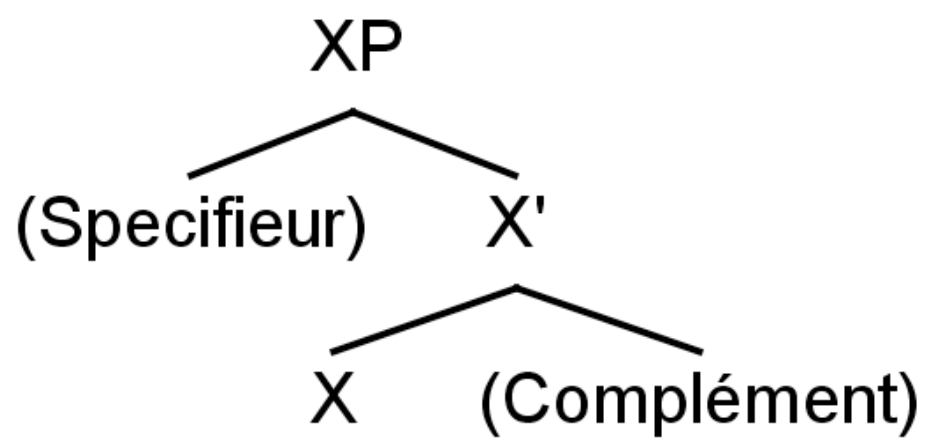

Fig. 2 


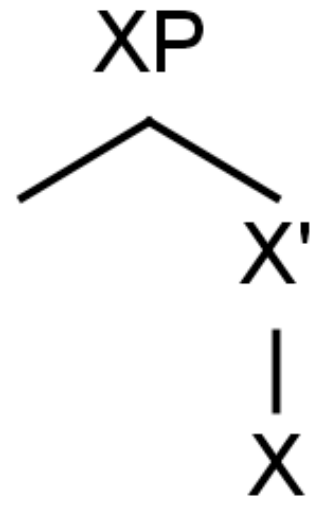

Fig 2'

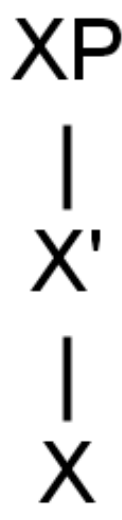


Fig. 2"

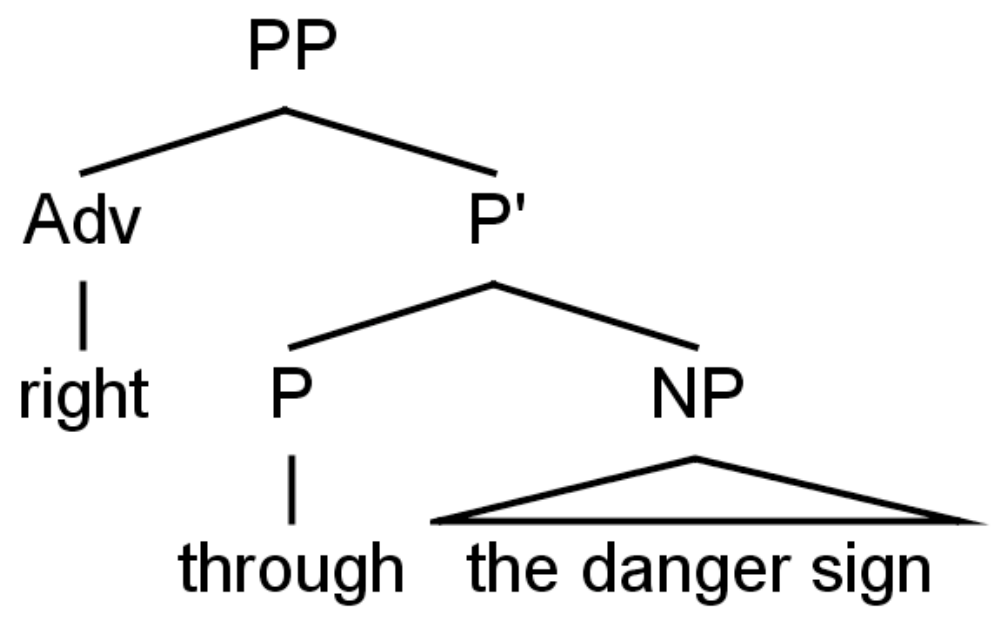

Fig. 3

9 Mais nous ne nous étendrons pas sur ce point ici. Revenons donc à ce que nous disions plus haut sur les catégories lexicales et fonctionnelles.

\section{Projections lexicales et projections fonctionnelles}

10 On illustrera ici l'articulation, en grammaire générative, entre les deux catégories. Soulignons tout d'abord ( $c f$. Figure 2) que toute tête est appelée à se projeter en un syntagme (projection maximale) en passant par une projection intermédiaire et que les têtes fonctionnelles ne font pas exception à cette règle de base. Par exemple, on a illustré en Figures 4 et 5 le parallélisme entre la projection fonctionnelle I(nflection), qui domine le VP, et la projection fonctionnelle D(eterminer), qui domine NP; sans entrer dans les détails, on entr'aperçoit ainsi le rôle de délimitation spatiale ou temporelle joué par le fonctionnel par rapport au lexical ${ }^{6}$. On pourra dire que, d'un côté, la flexion verbale appartient bien à une super-catégorie verbale, mais qu'elle se distingue du verbe par la présence d'un trait $\{+F\}$, de même pour $\mathrm{D}$ par rapport à $\mathrm{N}, \mathrm{V}$ et $\mathrm{N}$ se voyant de leur côté (cf. Fig. 1) affectés du trait $\{-F\}$. On aura donc, comme traits définitoires des catégories I et $D$ :

$$
\begin{aligned}
& \mathrm{I}=\{-\mathrm{N},+\mathrm{V},+\mathrm{F}\} \\
& \mathrm{D}=\{+\mathrm{N},-\mathrm{V},+\mathrm{F}\}
\end{aligned}
$$

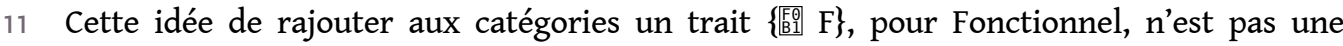
innovation récente, mais elle a été récemment systématisée dans une thèse (Littlefield 2006) dont nous dirons quelques mots dans la dernière partie de cette étude, et qui nous 
paraît pédagogiquement intéressante pour mieux fonder les distinctions indispensables dans le domaine prépositionnel.

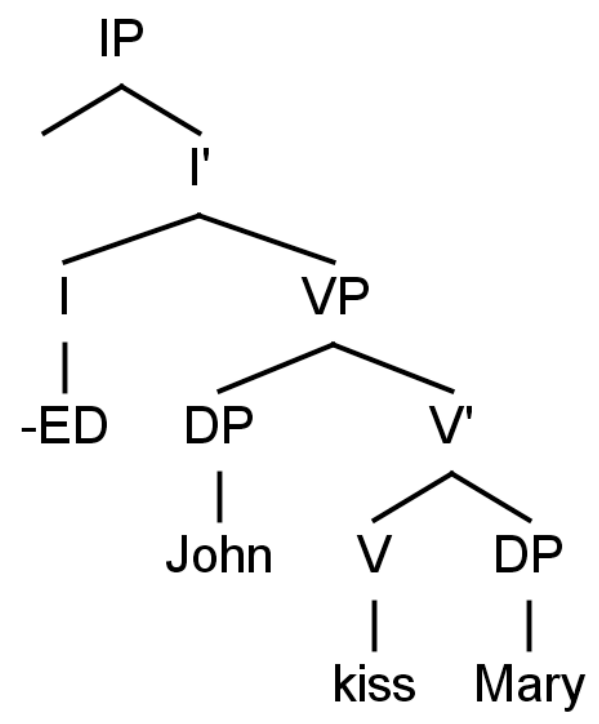

Fig. 4

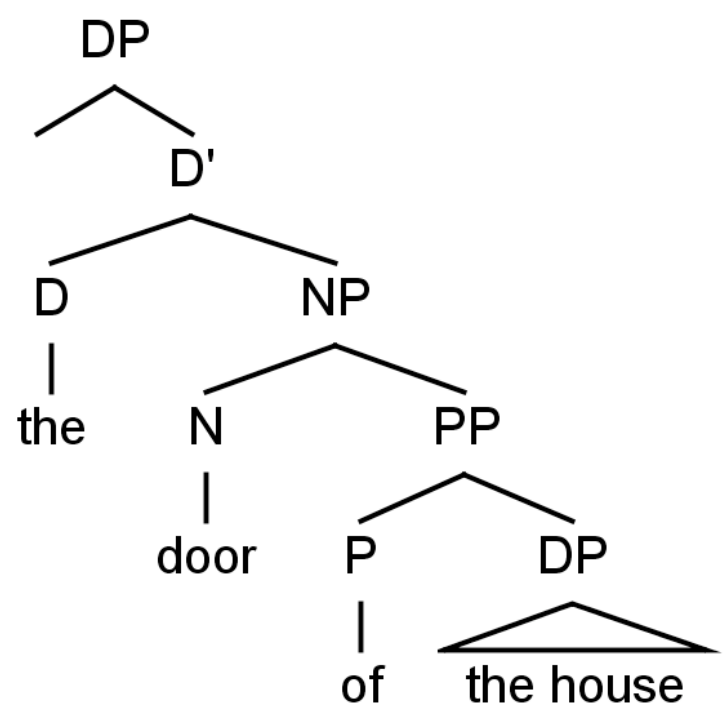


Fig. 5

\section{Prépositions et module casuel} des travaux ayant fondé la sémantique générative (Fillmore 1968, Anderson 1976), la théorie du cas devient un module à part entière dans la grammaire générative. Là encore, elle a connu de nombreux raffinements et de nombreuses sophistications jusqu'à aujourd'hui, mais nous nous bornerons, dans le cadre restreint de cette étude, à poser les grands principes. Le case filter («filtre du cas »), est un postulat central de la théorie, généralement formalisé par :

*[NP -case $]$

where NP is overt

Fig. 6

ce qui se lit à peu près comme ceci: tout groupe nominal ${ }^{7}$, s'il n'est pas phonologiquement nul, doit obligatoirement recevoir un cas, sous peine de rendre agrammatical l'énoncé dont il est un constituant. Il s'agit bien évidemment de cas abstrait, puisque, en anglais contemporain, à la différence par exemple de l'allemand contemporain, les cas ne sont plus morphologiquement visibles sur les groupes nominaux, seuls les pronoms les distinguent encore.

Or, on a bien précisé supra (cf. exemples [1]) que la préposition partageait avec le verbe la propriété d'assigner le cas à son complément. Mais peut-on dire que le cas assigné par verbe et préposition est le même? En apparence oui, (cf. Mary saw him / *he ; Mary came with him / 'he) et il s'agit de l'accusatif, cas objet. Mais si l'on observe les choses d'un peu plus près, en prenant un minimum de perspective historique, les choses ne sont pas si simples. Voici dans la première colonne un fragment de la déclinaison du pronom masculin en vieil-anglais (jusqu'au moyen-anglais) et, dans la deuxième colonne, la situation en anglais contemporain pour comparaison :
Nom he Nom he
Acc hine Acc him
Dat him Dat him
Gen his Gen his

On constate que la forme accusative originelle hine a disparu ; plus exactement, c'est le datif qui a absorbé l'accusatif, au point que la morphologie est désormais identique pour les deux cas. En anglais médiéval, les prépositions sont susceptibles de donner plusieurs cas: le datif pour la grande majorité d'entre elles, mais également, pour certaines, l'accusatif, et même parfois le génitif. L'anglais, il est banal de l'observer, est une langue avant tout germanique, et on observe les mêmes phénomènes en allemand contemporain, où certaines prépositions sont réputées « gouverner » tel ou tel cas. Par exemple, on sait qu'avec la même préposition, on aura des différences bien connues et lourdes de sens au plan de la syntaxe et de la sémantique. Ainsi :

i) Die Katze läuft unter den Tisch [the cat runs under the table]

ii) Die Katze schläft unter dem Tisch [the cat sleeps under the table]

En i), la préposition unter ( = under) gouverne l'accusatif, prototypiquement cas de l'objet direct, lui-même prototypiquement construit comme objet affecté. Sémantiquement, la 
table est affectée au sens où elle est au point d'arrivée ${ }^{8}$ d'une trajectoire : elle est donc construite comme une nouvelle localisation de l'entité en mouvement, celle-ci étant donc créditée d'un changement ${ }^{9}$. En revanche, en ii), la préposition assigne le datif, et la table est cognitivement construite comme non-affectée, ou minimalement affectée. Il n'y a pas de déplacement, le rôle sémantique associé sera « localisation » et non « but ».

On pourra, par conséquent, admettre comme hypothèse de travail que la préposition en anglais contemporain est bel et bien susceptible, même si morphologiquement ils demeureront impossibles à distinguer, d'assigner des cas différents à son complément. Nous y revenons plus bas. Par exemple, c'est le datif, et non l'accusatif, qui est assigné au DP complément de la préposition to dans la construction prépositionnelle à 3 arguments ci-dessous, représentée en VP-shell ${ }^{10}$; et c'est, dans les traitements classiques, entre autres ${ }^{11}$ parce que le même argument a perdu sa préposition rectrice qu'il doit remonter chercher un cas dans une position où il est gouverné par v :

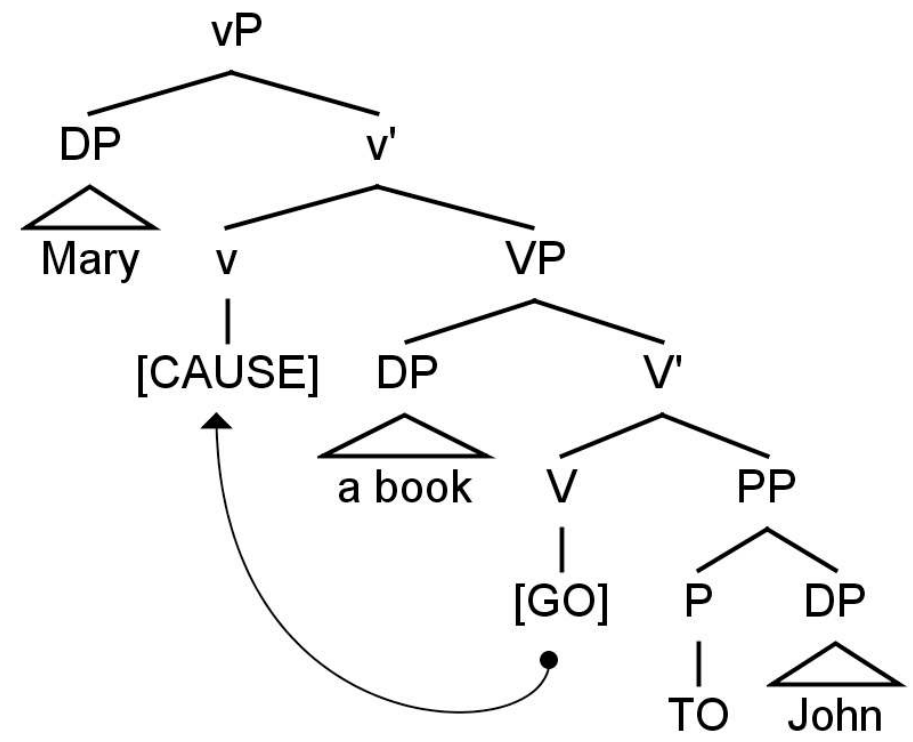




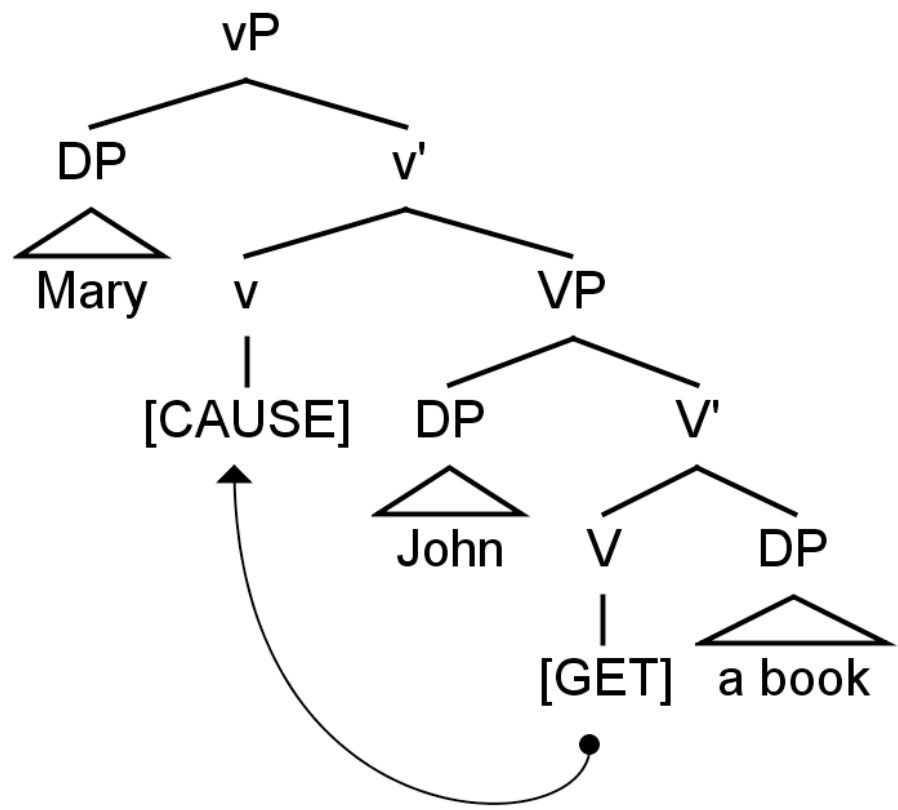

(Mary gave John a book)

Fig. 8

\section{Le domaine prépositionnel}

Regardons à présent comment nous pouvons intégrer les outils de ce modèle pour traiter, non seulement des prépositions centrales, mais de l'ensemble du domaine prépositionnel. C'est dire que nous suivrons ici bon nombre d'auteurs pour proposer un traitement unifié des prépositions, des adverbes (ou particules adverbiales, dans une terminologie également largement acceptée, cf. Bolinger 1971) et des particules. Nous tenterons également de montrer, avec notre boîte à outils, que la catégorie même des prépositions doit faire l'objet d'une distinction supplémentaire, ce qui nous donnera, au final, quatre sous-catégories dans le domaine.

\subsection{Quatre sous-catégories}

Commençons donc par les prépositions, dont on sait par des tests classiques qu'elles s'opposent en bloc aux adverbes et aux particules par le test de position :

[3a] Fill (up) the bucket (up) [particule]

[3b] Pick (up) my box (up) [adverbe]

[3c] Walk up the hill (*up [préposition]

[3d] Plant (*arefully) the seeds (carefully) [adverbe central]

Watch (*tomorrow) the movie (tomorrow) [adverbe central] 
Bring (*here) John (here) [adverbe central]

Hang (*askew) the picture (askew) [adverbe central] celui-ci n'est pas pronominal, évidemment...), ce qui n'est guère étonnant, l'anglais étant une langue à tête initiale (head initial language). Nous avons utilisé des exemples inventés pour montrer que UP, par exemple, peut relever des trois catégories à la fois, car dans notre texte, il n'apparaît que dans le rôle de particule illustré par [3a] : something she'd been locked up in (1. 25), She'd scoop us up (1. 42), the chicken she was cutting up (1. 43). Même si le test cité est loin d'être le seul proposé dans la littérature, il a l'avantage de fonctionner toujours et dans tous les cas. Ce qui ressort tout de même à l'évidence est que, si up est classé comme adverbe en [3b], les adverbes centraux utilisés en [3d], à titre de comparaison, n'ont pas le même comportement par rapport au test. Il existe d'autres tests syntaxiques pour distinguer les sous-catégories du domaine prépositionnel, sur lesquels nous reviendrons infra.

21 Mais demandons-nous d'abord si la classe des "vraies » prépositions est une classe homogène, en partant de l'interrogation que nous soulevions en introduction supra. Si nous nous donnons un tableau simplifié des propriétés distinguant traditionnellement les catégories fonctionnelles et lexicales, par exemple :

\begin{tabular}{|l|l|}
\hline Catégories lexicales & Catégories fonctionnelles \\
\hline - Contenu sémantique & - Absence de contenu sémantique « lourd » \\
- Classe ouverte & - Classe fermée \\
Inventaire limité & Inventaire limité \\
Création libre de nouveaux éléments & Pas de nouveaux éléments \\
- Type de complémentation peu contraint & - Type de complémentation très contraint \\
- Assignation de $\theta$-rôles & - Pas d'assignation de $\theta$-rôles \\
- Séparabilité d'avec complément & - Inséparabilité d'avec complément \\
- Indépendance morpho-phonologique & - Dépendance morpho-phonologique \\
\hline
\end{tabular}

nous constatons très vite que certaines prépositions vont fonctionner sans trop de problèmes avec les critères d'une des colonnes, mais pas avec d'autres :

- Certaines prépositions vont bel et bien, à l'instar des verbes, impliquer un contenu sémantique, et mettre en relation dans un « scénario » des objets, des entités du monde. C'est le cas classiquement des prépositions spatiales, in, on etc., qui, cognitivement, convoquent effectivement une situation, un événement (entendu au sens large de state of affairs), avec des participants ((in the) bureau drawer 1. 31, (on the) wheel 1. 11, (on the) white enamel kitchen table 1. 42).

- Certaines prépositions semblent bien disposer d'une assez grande latitude dans leur sélection de compléments, qui vont du DP, cas le plus classique (ink spilled in water, 1. 19, rigid with pain, 1. 17, at his office, 1. 35, etc.) au PP (pas d'exemples dans le texte, mais on peut imaginer the dog ran from under the table), mais également au CP (Ten days after the war ended, 1. 1, I was furious with Laura for what she'd done, 1.18 , from when we were little, 1 . $40)^{12}$, ou même au VP, si on analyse les gérondives ou les participiales comme tels (...with the policeman for implying that she'd done it, 1. 18). 

explorée depuis très longtemps en syntaxe et la plupart des analyses des adjoints locatifs (du type The students drank beer in class, ou dans notre texte a hot wind was blowing around my head, 1. 19), bénéfactifs (She bought a book for her daughter), de trajectoire (The boy threw the ball into the kitchen) convergent pour faire de la préposition et non du verbe l'assignateur du $\theta$-rôle de son complément.

- Pour ce qui est de la séparabilité, on peut penser à celle, bien étudiée, des compléments prépositionnels sous mouvement- $W H$; nous en avons quelques exemples dans le texte avec something she'd been locked up in (1. 25) ou What had she been thinking of (1. 28); cette séparabilité se vérifie d'ailleurs également sous clivage (It was the table that the dog ran under) ou pseudo-clivage (What the dog ran under was the table).

che, on vérifiera que l'on peut trouver des arguments tout aussi concluants, mais dans l'autre sens, avec d'autres prépositions. Ainsi, même si la grammaire traditionnelle en compte 50 à 60, même si certaines études de corpus en recensent jusqu'à 200, il n'en reste pas moins qu'elles constituent une classe fermée. Par ailleurs, le contenu sémantique de certaines prépositions dites «incolores» (Spang-Hanssen 1963, Cadiot 1997), ou "désémantisées» est, par rapport à d'autres, plutôt voisin de zéro. Le représentant prototypique de ce cas de figure est bien évidemment $O F$, qui est la plus fréquente dans notre texte, ce qui n'est guère surprenant dans la mesure où elle est aussi la plus fréquente des prépositions en corpus ${ }^{13}$. Par ailleurs, les analyses syntaxiques contemporaines convergent pour dire que cette même préposition n'assigne pas de $\theta$ rôle. Dans les exemples bien connus comme the destruction of the city (by the enemy) ou Mary is jealous of John, c'est bel et bien le nom ou l'adjectif qui assigne les deux rôles (agent / patient dans un cas, expérient / source dans l'autre). Bon nombre des of de notre texte, par exemple, exprimant des relations sémantiques de type partie-tout, peuvent être analysées ainsi, avec des rôles de type thème / localisation (Chunks of the bridge 1. 4, the strands of my hair 1. 19, a lump of brown sugar 1. 44); on ne s'étonnera donc guère que la plupart des syntacticiens, depuis les années 70 au moins, conviennent que of assigne le génitif, et non l'accusatif ou le datif ${ }^{14}$. Manifestement donc, la classe n'est pas homogène (ce qui n'est nullement une nouveauté pour les spécialistes de la question, bien évidemment).

Nous tenons donc (cf. Littlefield 2006), à partir de cette première opposition entre ces prépositions au contenu sémantique voisin de zéro et les prépositions à contenu plus lourd, l'amorce d'un système plus ambitieux de description du «domaine prépositionnel ». Les premières se verront attribuer un trait $\{-\mathrm{L}\}$ (ce qui signifie qu'elles n'ont pas de valeur lexicale), et $\{+F\}$ (le trait «fonctionnel» dont il a été question plus

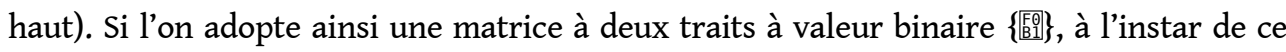

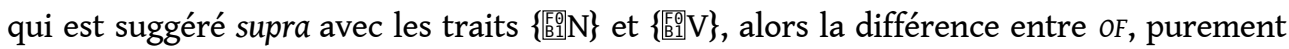
fonctionnel, et les prépositions à contenu sémantique sera que ces dernières prennent tout naturellement la valeur $\{+\}$ pour le trait lexical. C'est pour cela que, dans les approches de type continuum (qui remontent au moins à Ross 1972), on les range parfois dans la catégorie des constituants "semi-lexicaux ", c'est-à-dire ceux qui ont à la fois un contenu lexical et une valeur fonctionnelle. C'est le sens de la Figure 9 ci-dessous ${ }^{15}$ : 


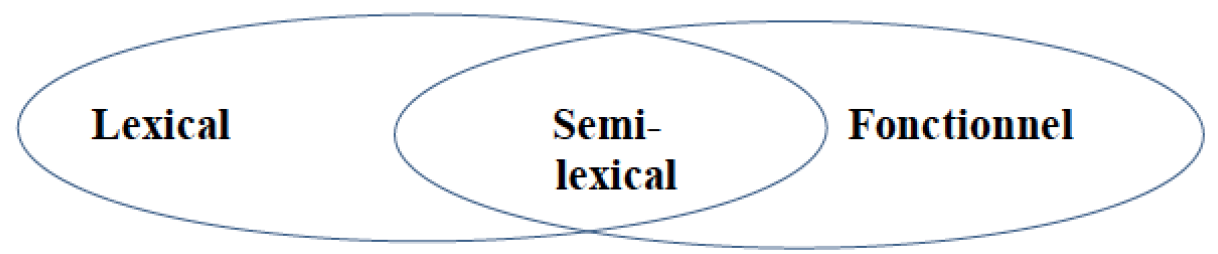

Fig. 9

\section{$\{-F,+L\}$ et $\{-L,-F\}$, qui correspondent aux quatre types que nous avons déjà évoqués supra,} et que nous résumons dans le tableau ci-dessous, sur le modèle de la Figure 1 supra :

\begin{tabular}{|l|l|l|}
\hline & $\{\mathbf{F}\}$ & $\{\mathbf{L}\}$ \\
\hline Préposition fonctionnelle & + & - \\
\hline Préposition semi-lexicale & + & + \\
\hline Adverbe & - & + \\
\hline Particule & - & - \\
\hline
\end{tabular}

Fig. 10

On vérifiera, si l'on regroupe les catégories deux par deux, que les tests syntaxiques précédemment évoqués, et d'autres que nous en profitons pour signaler, vont bien séparer les choses. Par exemple, les deux catégories partageant le trait $\{+\mathrm{F}\}$ admettent le pied-piping (préposition semi-lexicale: Up which hill did they run? ou préposition fonctionnelle: Of whose performance was Mary proud), mais pas les autres (particule: *Up whose number did she look?, ou adverbe : *Apart which toy did she pull ?)

On peut également regrouper adverbes et prépositions semi-lexicales, c'est-à-dire les catégories partageant le trait $\{+\mathrm{L}\}$. Là, nous retombons sur la propriété bien connue, déjà évoquée au tableau de la Figure 10, de liberté vs contrainte sur la compositionnalité. Autant la liberté combinatoire est très grande avec adverbes et prépositions semilexicales (The girl pushed the toy around / across / over, The boy walked in / into / across / by the park), autant bien entendu elle est restreinte, voire nulle, avec particules ou prépositions fonctionnelles : they ate up (*down) the pizza / she is proud of $\left({ }^{*}\right.$ to) her fiancée. On peut également constater que la modification par un adverbe de degré, illustrée à la Figure 3 et par l'exemple she went right through the Danger sign, est 
strictement impossible avec une préposition fonctionnelle (*Mary is proud right of her dog), et l'on retrouve le contraste entre she pulled the toy straight apart et *She looked the number straight up. Autrement dit, prépositions semi-lexicales comme adverbes auront un spécifieur dans leur structure, mais pas la particule ni la préposition fonctionnelle.

Regardons à présent les choses en détail à partir de notre corpus.

\section{Les prépositions fonctionnelles $(\{+F,-L\})$}

32 Les prépositions de cette catégorie, avec of comme exemple prototypique, sont bien connues et bien décrites comme porteuses d'un poids sémantique très faible, voire nul. On les qualifie souvent, d'ailleurs, de "vides » (dummy), ou de simples outils assignateurs de cas. Nous ne reviendrons pas sur les exemples donnés supra avec $O F$, auxquels on peut rajouter des énoncés comme toute la série commençant par What had she been thinking of (1. 28), où c'est bel et bien le prédicat verbal qui assigne le rôle sémantique et non la préposition, mais également des cas non représentés dans le texte, où c'est cette fois un prédicat adjectival qui assigne ce rôle (Mary is proud * (of) her dog; They are disdainful *of) the students). On pourra traiter de la même façon, d'ailleurs, des exemples tels I was informed of the accident (1. 6), mais également She was completely ruthless in that way (1.14), in a sober colour (1. 23) ou encore I must be in shock (1. 38). Car, même si of est bel et bien l'exemple prototypique, d'autres prépositions peuvent également jouer ce rôle de simples marqueurs de compléments de noms (the academy's support *(for / of) the proposal; his capacity*(for) hope ; her desire*(for) chocolate, etc.), d'adjectifs (Bill is keen *(on) soccer; She is angry *(at / with) him ; They are good *(at) volleyball, etc.), ou bien évidemment de verbes ( She clings * (to) her faith / *She clings; The students pore *(over) their notes before the exam /*The students pore; The inventor toyed *(with) the idea / ${ }^{*}$ The inventor toyed). Les verbes cités nécessitent tous un argument interne, comme le montrent les manipulations proposées. Dans notre texte, il est assez clair que les deux WITH de la phrase 1.17-18: I was furious with Laura for what she'd done, but also with the policeman for implying that she'd done it peuvent relever de cette catégorie, dans la mesure où c'est bien le prédicat adjectival be furious qui assigne les rôles sémantiques à ses arguments.

On réservera un traitement particulier aux agentive BY-phrases dans les structures passives, dont on avait un exemple 1. 6: I was informed of the accident by a policeman. Pour aller vite, le complément de la BY-phrase étant à l'origine l'argument externe de la structure active correspondante ( = the policeman informed me), on pourrait dire que c'est le verbe qui assigne le rôle sémantique agent à travers la préposition. Cependant, nous suivrons ici une analyse fort courante, qui tient que l'une des fonctions essentielles du passif est précisément d'effacer le rôle sémantique externe assigné par le verbe. C'est d'ailleurs la raison pour laquelle la BY-phrase est complètement optionnelle dans les passifs, puisqu'on sait pour l'anglais que $90 \%$ de ceux-ci sont précisément des short passives (I was informed of the accident\#). De plus, la préposition $B Y$ est susceptible d'assigner le rôle sémantique d'agent dans d'autres constructions non-passives (the painting by Monet , the song by Dylan, shot by Messi, etc.). Ce qui nous conduit plutôt à voir dans BY une préposition semi-lexicale, ayant à la fois la propriété d'assigner un rôle sémantique et celle d'assigner le cas, et nous fournit une parfait transition vers cette catégorie. 


\section{Les prépositions semi-lexicales $(\{+\mathrm{F},+\mathrm{L}\})$}

34 Ces prépositions, les plus prototypiques de la catégorie, assignent le cas (trait $\{+F\}$ ), et par conséquent peuvent être représentées comme transitives : elles appellent un complément interne (cf. supra Figure 2). Et de plus (\{trait $+\mathrm{L}\}$ ), elles portent un contenu sémantique descriptif, car toutes expriment, comme nous le mentionnions supra, des scénarios, des relations : elles localisent des objets par rapport à un repère. Nous n'irons pas plus loin dans ces considérations sémantiques, dont les autres contributions au présent collectif se font largement l'écho, et nous tournerons plutôt vers les questions de rôles sémantiques que nous avons déjà largement abordés. En l'occurrence, le rôle le plus souvent associé aux prépositions semi-lexicales est très probablement le locatif, ce qui n'est guère surprenant si l'on reprend ce que nous disions à l'instant sur leur rôle de localisateurs par rapport à des repères. Pour des raisons narratives assez évidentes, ce type de prépositions est assez fréquent dans notre texte, citons-en quelques exemples: the shallow creek at the bottom (1. 4),...in the bureau drawer where I kept my stockings (1. 31), He said the tires may have caught on a streetcar track (1. 7), They'd noticed her hands on the wheel (l. 11), sit us on the white enamel kitchen table (1.42) ou encore les trois occurrences de off (1. 1, 10 et 28). D'autres prépositions de ce type assignent des rôles tels but (typiquement To, dans les exemples comme he went to Paris, dont nous avons vu qu'ils étaient absents de notre texte, source (from a distance, 1. 16) ou trajectoire (path), par exemple she went right through the Danger sign (1. 2), a hot wind was blowing around my head (1. 19) ou encore, bien évidemment, les quatre occurrences de INTO du texte: The car fell a hundred feet into the ravine (1. 2), ...rolled down into the shallow creek (1. 3), I went into my dressing-room (1. 36) ; la quatrième occurrence, burst into flames (1. 3), n'est dans le cadre de la présente analyse guère différente, même si au plan sémantique la trajectoire est métaphorique et non seulement physique, et que nous sommes à la limite des constructions résultatives.

On pourra, dans cette section, s'attarder quelque peu sur le cas de WITH, très épineux en raison de la gamme des rôles sémantiques qu'elle est susceptible d'assigner. Nous avons déjà vu supra que deux occurrences de cette préposition relevaient davantage de la catégorie des prépositions fonctionnelles ; mais il y en a cinq autres dans notre texte qui, quant à elles, sont assez clairement semi-lexicales et assignent divers rôles. Le cas qui vient le plus facilement à l'esprit est bien entendu celui d'instrument (He cut the steak with a knife), absent de notre texte, mais on peut également citer la manière, dont relève par exemple ...had plunged off the bridge with no more fuss than stepping off a curb (1. 10), quelque chose que l'on pourrait nommer, faute de mieux, accompagnement, que l'on peut définir comme une relation de modification, en général paraphrasable par having, que l'on retrouve dans $a$ hat with a veil (1.34). Et on ajoutera enfin quelque chose qui ressemble singulièrement à la cause, assez facilement repérable dans feathery with new leaves (1.3) et rigid with pain (1. 17).

On aura bien entendu compris que, au plan purement syntaxique, la préposition, qu'elle soit lexicale ou fonctionnelle, forme avec son complément un constituant, et que l'ensemble est à son tour complément du verbe, ou éventuellement adjoint si le verbe en question est intransitif. On a représenté ci-dessous les deux cas à partir de deux exemples cités supra : 


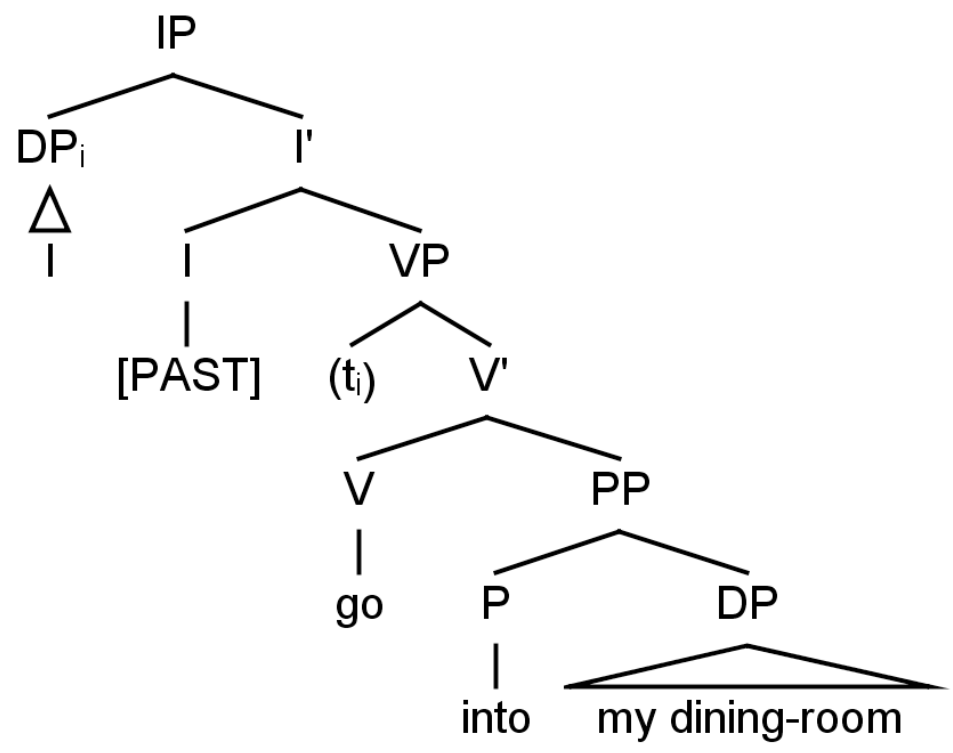

Fig. 11

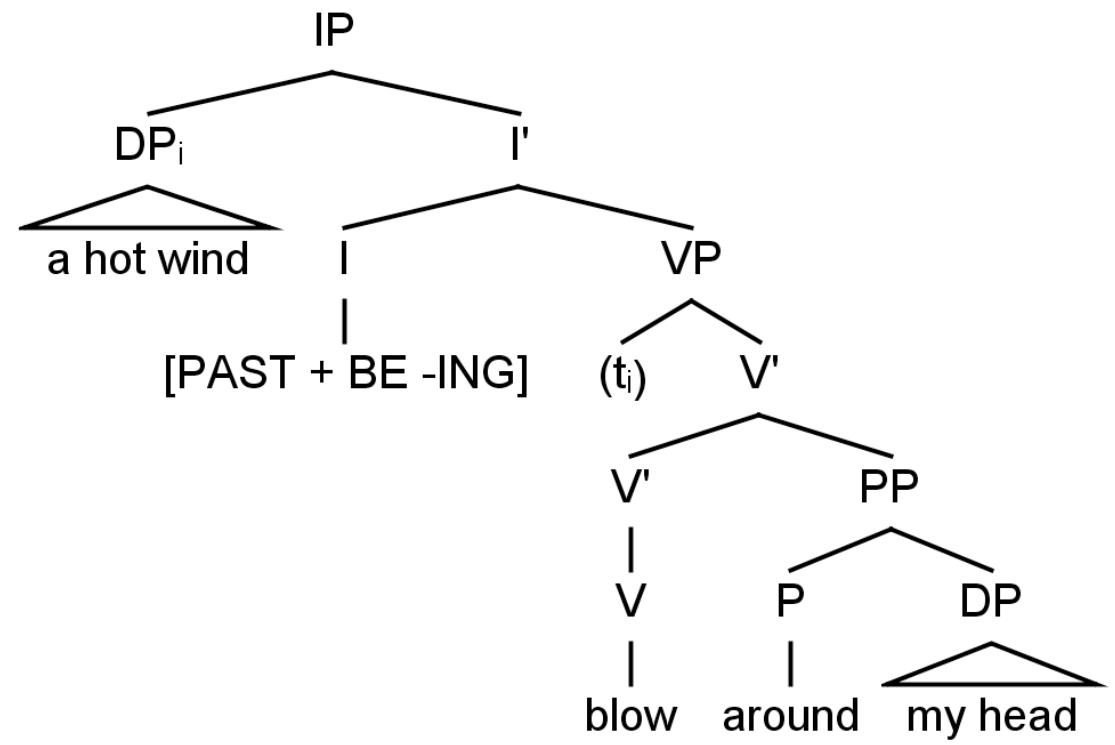

Fig. 12 


\section{Les adverbes $(\{-F,+L\})$ et les particules $(\{-F,-L\})$}

37 Passons à présent aux deux sous-catégories du domaine prépositionnel qui partagent le trait $\{-\mathrm{F}\}$ : étant non-fonctionnelles, elles devront nécessairement être représentées comme intransitives (cf. supra Figures 2' et 2"). On assiste depuis des décennies à un débat très vif entre syntacticiens, d'où il ressort tout de même que les deux sous-catégories présentent un degré important de chevauchement, et semblent se fondre l'une dans l'autre, avec des cas intermédiaires indécidables. D'où notre parti-pris de traiter les deux en même temps en insistant sur ce qui, globalement, les oppose aux prépositions fonctionnelles ou semi-lexicales, à savoir en l'occurrence le trait $\{-F\}$, et qui, cela ressort également des travaux des deux dernières décennies, amène une majorité d'auteurs à leur attribuer deux projections bien séparées, ce que nous détaillerons un peu plus loin. Mais commençons par citer les tests classiques distinguant adverbes et particules, par exemple quelques-uns proposés par Fraser (1976) :

[4a] Her pulling (up) of her sleeve (up) was unnecessary

[4b] His looking up of the number (*up) was unnecessary

[5a] Sue pulled her sleeve up, and Sam, his pant leg up

[5b] *He looked the number up, and Tim, the address up

[6a] Sue pulled her sleeve only part of the way / all the way up

[6b] ${ }^{*}$ Tom looked the fact only part of the way / all the way up

[7a] Sue pulled her socks UP, not DOWN

[7b] *Tom looked the information UP, not OVER

Bien sûr, les énoncés obtenus, même donnés pour grammaticaux, ne sont pas des modèles de naturel, c'est en général le reproche fait aux manipulations syntaxiques... Mais quoi qu'il en soit, pour chaque paire, on constatera que l'adverbe passe les différents tests avec succès (nom verbal en [4], gapping en [5], modification par adverbe de degré en [6], accent contrastif en [7]), mais que la particule donne toujours des séquences agrammaticales. Ces tests ont fait l'objet de critiques et de reformulations dont il serait trop fastidieux de donner la liste ici (pour une excellente synthèse, voir Cappelle 2005). Mais tentons de reprendre les choses en détail à partir des exemples de notre texte.

Les adverbes, catégorie purement lexicale, ont une contribution sémantique substantielle, mais ne relient pas d'éléments entre eux à travers l'assignation du cas ; ils n'assignent pas non plus de rôle sémantique. À l'instar des prépositions semi-lexicales, leur sens est généralement spatial. Bolinger (1971), note que les adverbes (qu'il nomme adverbial particles) must contain two features, one of motion-through-location, the other of terminus or result. Cela est d'un intérêt extrême pour la sémantique et la linguistique cognitive, car si nous interprétons, cela signifie que l'adverbe va prédiquer quelque chose du DP, généralement une nouvelle localisation de ce dernier, se situant au point d'arrivée de la trajectoire qu'implique l'événement dénoté par le verbe. Nous ne signalons ces faits bien connus que pour leurs implications syntaxiques, que nous abordons infra. Mais regardons les exemples du texte et voyons comment ils illustrent les quelques propriétés que nous venons d'énumérer. On citera en particulier deux occurrences de Down, celle de la 1. 3 (... and rolled down into the shallow creek at the bottom), sur laquelle nous reviendrons, et celle de la l. 15 (I'll come down as soon as I can); on a également deux occurrences de OUT, celle de la 1.16 (I could barely get the words out) et celle de la 1.43 (the pie dough she was rolling out); et une de OVER 1. 38 (I was cold all over), où all est manifestement en position de spécifieur. Pour ce qui concerne la catégorie des particules, selon les quelques critères que nous nous 
sommes donnés, nous aurions donc dans notre texte, outre les trois occurrences de UP cités supra, deux occurrences de Down (qui se ramènent en fait à une seule, puisqu'il y a écho): Just calm down and show me where (1. 45) et They can't calm down (1. 46).

D'un point de vue statistique, il est peut-être intéressant de noter que OFF, DOWN, OUT et UP, tous présents dans notre texte, ont des emplois bien plus fréquents comme adverbes que comme prépositions, à la différence par exemple de on et IN : dans le British National Corpus (BNC), OFF compte 37751 usages adverbiaux pour 8060 prépositionnels, DOWN 72709 adverbiaux pour 5182 prépositionnels), OUT 145706 pour 607 et UP 158,064 pour $3424^{16}$. Notons tout de même que le BNC ne distingue pas dans son codage entre ce que nous nommons "adverbe » et ce que nous nommons " particule ", mais redisons encore que ces deux sous-catégories partagent un trait commun, celui d'être $\{-F\}$, l'opposition intéressante étant par conséquent celle, binaire, entre prépositions (fonctionnelles ou semi-lexicales) d'un côté et adverbes / particules de l'autre.

Regardons donc de plus près, pour terminer ce tour d'horizon, la syntaxe des adverbes et des particules; dans cette présentation très généraliste, nous n'entrerons pas dans des détails trop techniques, mais en gros, on peut distinguer deux grandes tendances: celle qui propose qu'adverbes ou particules sont générés avec le verbe en structure profonde, formant dès le départ un verbe complexe, et celle, plus fructueuse à nos yeux, de la small clause («proposition réduite ») ou de l'incorporation (Kayne 1985, den Dikken 1995), où la particule ${ }^{17}$ est générée comme tête de son propre syntagme (PrtP = Particle Phrase), et peut s'incorporer au verbe dans un second temps. C'est le sens de la Fig. 13 ci-dessous, où nous reprenons, légèrement adapté, l'exemple de la 1. 43 de notre texte (the chicken she was cutting up), en ne représentant que la partie pertinente pour ne pas alourdir la lecture :

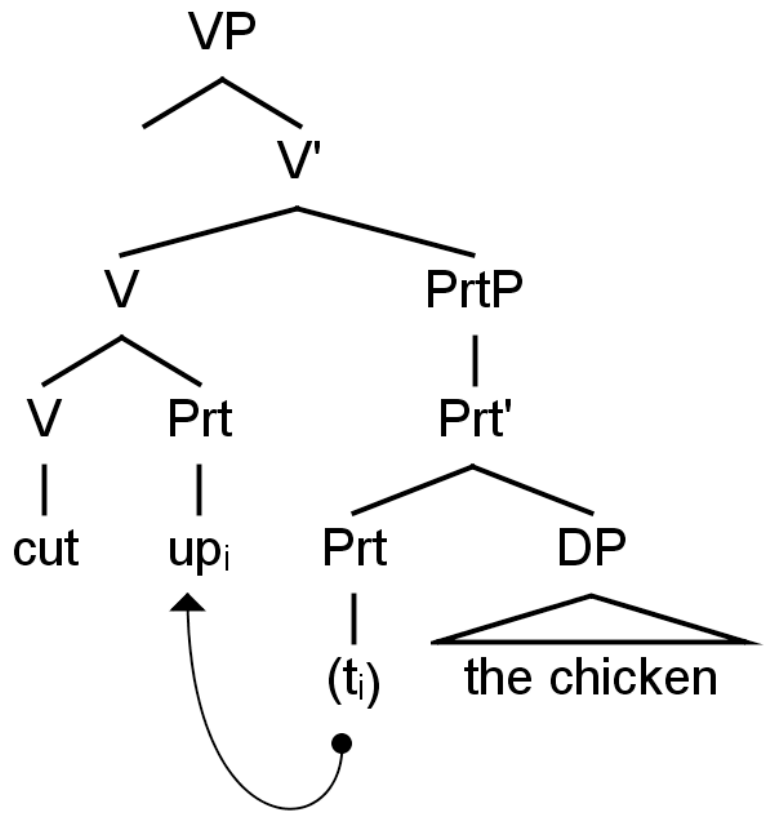


Fig. 13 complément du verbe cut, vient s'incorporer à ce verbe pour former un verbe complexe. Sans entrer dans tous les détails de la théorie, c'est le filtre du cas (cf. supra § 3) qui va motiver une partie des mécanismes en jeu. En raison du déplacement de up, la PrtP se retrouve sans tête et est alors dite "transparente", permettant au verbe complexe nouvellement formé, cut up, d'assigner le cas accusatif au DP the chicken, qui va satisfaire ainsi au filtre du cas. Si la particule restait in situ dans sa position de tête du syntagme, le verbe ne pourrait pas assigner le cas à travers elle, de même qu'un verbe ne peut assigner le cas à travers une préposition ${ }^{18}$, c'est la préposition elle-même qui assigne le cas à son complément (par exemple en Figure 11).

Souvenons-nous à présent du test présenté supra en [3] : les prépositions, contrairement aux adverbes et aux particules, ne peuvent jamais être séparées du verbe par l'objet, ce qui interdisait *Walk the hill up en [3c], ou pour reprendre n'importe lequel de nos exemples du texte, ${ }^{*}$ I went my dressing-room into, ou, pour les prépositions fonctionnelles, * she had been thinking Alex of. En revanche, cut the chicken up, roll the pie dough out, etc., sont parfaitement standard. Nous avons représenté ci-dessous cette configuration pour comparaison avec la Figure 13:

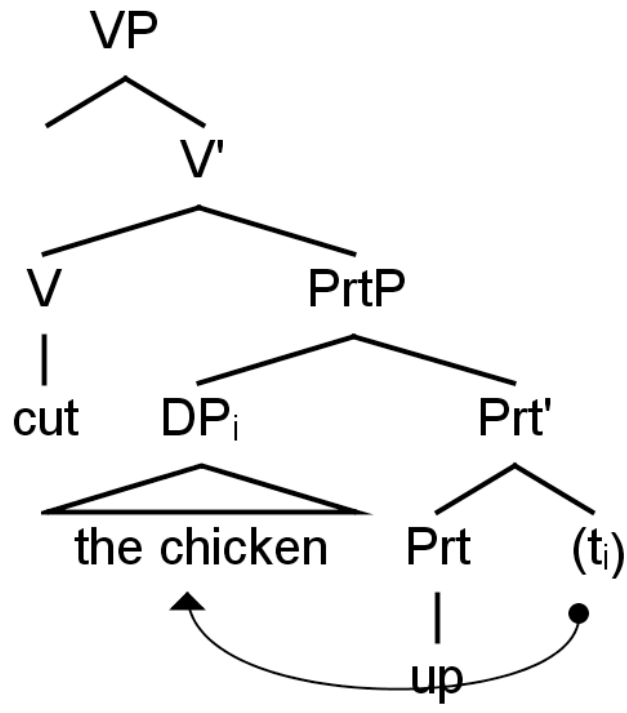

Fig. 14

Comme on pourra facilement le constater, la situation de départ est la même qu'en Figure 13, mais cette fois, la particule ne s'est pas déplacée. La conséquence est que, comme nous venons de le montrer, le verbe ne peut assigner l'accusatif, laissant le DP dans une situation de violation du filtre du cas, et le contraignant à se déplacer dans la position de spécifieur de la PrtP, devenant ainsi adjacent au verbe qui dès lors lui assigne l'accusatif. 
N'oublions pas non plus que la configuration de la Figure 14 est la seule disponible pour les pronoms (she cut it up $/{ }^{*}$ she cut up it), pour des raisons très complexes que nous ne développerons pas ici; de même que, pour des raisons sans doute plus claires intuitivement, elle serait agrammaticale dans le cas où le DP serait plus lourd (*she cut the chicken that she had bought at Walmart's the day before up).

Notons tout de même qu'il existe dans la littérature un certain nombre de solutions alternatives à celle-ci, dans lesquelles c'est le verbe lui-même qui se déplace, soit vers une projection aspectuelle AspP située juste au-dessus du VP (par exemple Felser 1998), soit vers la projection V dans une optique de slit-VP (par exemple Radford 1997, 2009). Cette dernière approche est d'ailleurs la traduction syntaxique des propriétés sémantiques des adverbes et dans une moindre mesure (certaines étant tout de même plus ou moins incolores) des particules que nous rappelions supra avec Bolinger (1971).

Nous terminerons par l'examen de quelques cas qui montrent que les choses peuvent être en fait bien plus complexes qu'il n'y paraît. Revenons par exemple sur celui de la l. 3, déjà cité supra, mais que nous répétons ici : ...and rolled down into the shallow creek at the bottom. Dans la suite down into, il paraît assez clair, au vu des analyses précédentes, que down est adverbe (si l'on s'en tient à nos distinctions précédentes adverbe/particule), et que into est préposition semi-lexicale. On peut également traiter de la même façon something she'd been locked up in (1. 25), où up est particule et in préposition semi-lexicale. Reste à se demander quelle sera la syntaxe de ces deux énoncés, autrement dit comment, par rapport aux représentations des Figures 13 et 14, vont s'articuler l'une à l'autre les syntagmes PrtP et PP, dont il apparaît clairement, dans les analyses contemporaines, qu'ils sont différents. On sait aussi depuis bien longtemps (cf. par exemple Svevonius 1996, Jackendoff 2002, Cappelle, op. cit.) que, dans ce type de configuration, la particule ne saurait apparaitre à droite du syntagme prépositionnel : they locked up the man / the locked the man up, mais they locked up the man in a room /*they locked the man in a room up, et qu'il ne s'agit pas ici de questions de lourdeur du constituant. Que PrtP et PP soient deux syntagmes différents est assez facile à corroborer par des manipulations assez simples, comme en [8] ci-dessous ${ }^{19}$ :

[8a] House prices have [gone up] [by more than $50 \%$ ].

[8b] House prices have [risen] [by more than $50 \%]$.

[8c] House prices have [gone up] [dramatically].

Là, on peut voir que l'on peut substituer ([8b]) un verbe simple au verbe complexe en laissant le syntagme prépositionnel inchangé, ou au contraire ([8b]), substituer un autre syntagme à ce syntagme prépositionnel, en laissant cette fois inchangé le verbe complexe.

En fonction de cette analyse, nous avons représenté le premier exemple ci-dessous en Fig. 15 , en simplifiant le DP complément pour le rendre plus lisible : 


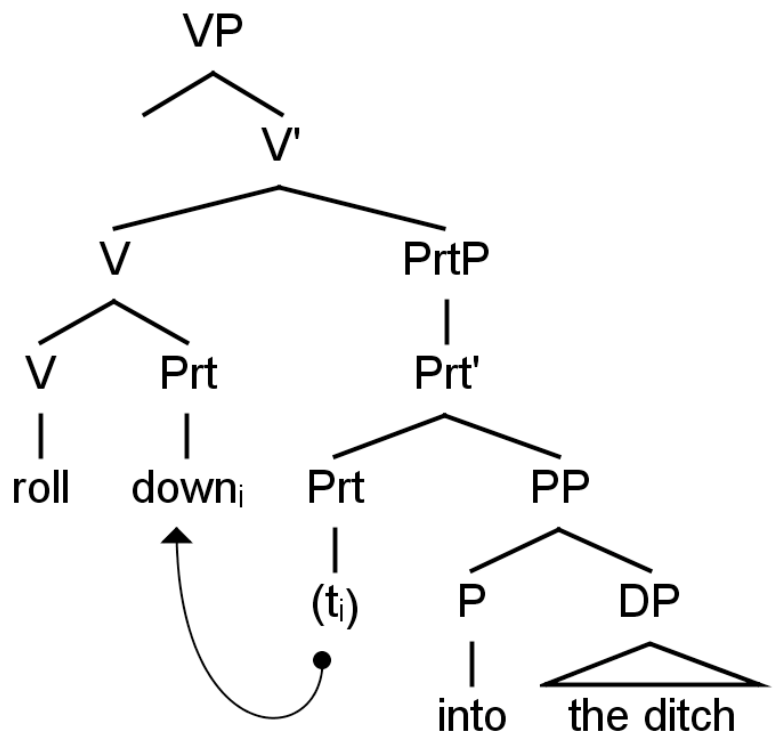

Fig. 15

49 C'est dire que la particule, ou l'adverbe, prend en structure profonde pour complément le syntagme prépositionnel, ce qui semble aller à l'encontre des intuitions (prépositions = transitives, adverbes/particules = intransitives) ; ce n'est que dans un second temps, comme on l'a expliqué supra, qu'elle va quitter sa position pour aller s'incorporer au verbe.

50 Mais les choses sont bien plus complexes que cela, car des exemples (absents de notre texte) comme

[9a] Up on the wall hung some of the most beautiful canvases I've ever seen.

51 ne sont pas justiciables de la même analyse. En effet, on peut montrer par quelques manipulations simples que cette fois, contrairement aux exemples en [8], la particule peut être effacée sans que la grammaticalité de la phrase ([9b]) et que, au contraire, l'énoncé devient agrammatical si c'est la préposition qui est effacée ([9c]) :

[9b] On the wall hung some of the most beautiful canvases I've ever seen.

[9c] *Up hung some of the most beautiful canvases I've ever seen.

Dans de tels exemples, fréquents dans les inversions locatives, c'est la particule qui (cf. supra Fig. 3) est en position de spécifieur du PP, comme nous le représentons ci-dessous, ce qui est un cas de figure très fréquent, pas seulement avec des $\mathrm{PP}$, mais par exemple avec des adverbes (over there, out yonder, etc.) : 


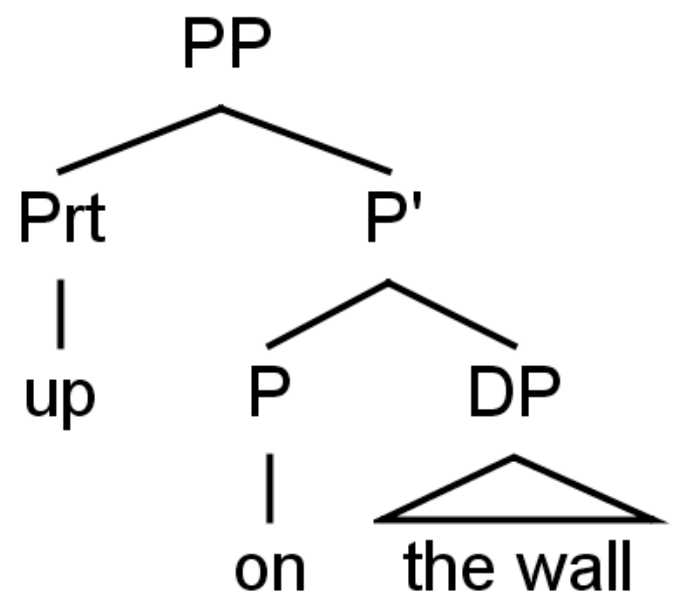

Fig. 16

Et il existe encore bon nombre de cas de figure différents, non représentés dans notre corpus, et que nous ne pouvons pas passer en revue ni même évoquer dans le cadre restreint de la présente étude.

\section{Conclusion}

54 Nous nous sommes efforcé, dans le cadre de la présente étude, de séparer autant que faire se peut les questions de syntaxe des questions de sémantique, mais il n'en reste pas moins, dans notre esprit, qu'elles ne peuvent qu'être étroitement liées. Nous avons tenté de montrer qu'on pouvait traiter, plutôt que des prépositions, de ce que l'on a nommé le domaine prépositionnel, et que ce domaine pouvait, sur la base de traits binaires, être structuré en quatre sous-domaines dotés à la fois de propriétés distinctes et de propriétés communes. Les points-clés, au plan de la syntaxe, sont la capacité à assigner ou non des rôles sémantiques, la capacité à assigner ou non un cas, lequel n'est pas, comme on le dit trop souvent (et comme dans le cas du verbe, qui assigne uniquement l'accusatif), uniforme. Et on a essayé de montrer également que les projections sont différentes dans le cas des prépositions d'un côté, des adverbes ou particules de l'autre, qu'elles avaient des propriétés syntaxiques différentes, et également qu'elles pouvaient se combiner dans un ordre bien précis. Il reste encore, malgré la vaste littérature sur ces questions, bien des points flous ou mal expliqués, nous espérons simplement avoir un peu contribué à clarifier les choses. 


\section{BIBLIOGRAPHIE}

Anderson, J. (1976). The Grammar Of Case : Towards A Localistic Theory, Cambridge : CUP.

Arnauld A. et Lancelot C. (1660). Grammaire générale et raisonnée. (Editions Allia, 2010).

Baker, M. (2003). Lexical Categories : Verbs, Nouns, and Adjectives, Cambridge : C.U.P.

Baker, M. (1997). 'Thematic Roles and Syntactic Structure', in L. Haegeman (ed.), Elements of Grammar, Dordrecht : Kluwer, 73-137.

Beavers, J. (2011). 'On Affectedness', in Natural Language and Linguistic Theory, 29 (2).

Bolinger, D. (1971). The Phrasal Verb in English, Cambridge : MIT Press.

Cadiot, P. (1997). Les prépositions abstraites du français, Paris : Armand Colin,

Cappelle, B. (2005). Particle Patterns in English - A Comprehensive Coverage, PhD Thesis, University of Leuwen.

Chomsky, N. (1965). Aspects of the Theory of Syntax, Cambridge : MIT Press.

Chomsky, N. (1970), 'Remarks on Nominalization', in R. Jacobs and P. Rosenbaum (eds), Readings in English Transformational Grammar, Waltham, MA : Ginn,184-221.

den Dikken, M. (1995). Particles: On the syntax of verb-particle, triadic, and causative constructions, Oxford : O.U.P.

Emonds, J. (1985). A unified theory of syntactic categories, Dordrecht : Foris.

Felser, C. (1998). 'Perception and Control : A Minimalist Analysis of English Direct Perception Complements', in Journal of Linguistics, vol. 34 (2), 351-385

Fillmore, C. (1968). 'The Case for Case', in Bach \& Harms (eds), Universals in Linguistic Theory. New York : Holt, Rinehart, and Winston, 1-88

Fraser, B. (1976). The Verb-particle Construction in English, Cambridge : MIT Press.

Jackendoff, R. (1977). X-bar Syntax - A Study of Phrase Structure, Cambridge, MIT Press.

Jackendoff, R. (2002). 'English particle constructions, the lexicon, and the autonomy of syntax', in N. Dehé, R., Jackendoff, A. McIntyre \& S. Urban, Verb-Particle Explorations, Berlin, New York, Mouton de Gruyter.

Jespersen, O. (1924). The Philosophy of Grammar, London : George Allen and Unwin

Kayne, R. (1985). 'Principles of particle constructions', in J. Guéron, G. Obenauer, \& J-Y. Pollock (eds), Grammatical representation, Dordrecht : Foris, 101-140.

Khalifa, J.-C. (2004). Syntaxe de l'anglais. Théories et Pratique de l'énoncé complexe, Gap : Ophrys.

Littlefield, H. (2006). Syntax and Acquisition in the Prepositional Domain : Evidence from English for finegrained syntactic categories, PhD Dissertation, Boston University.

Radford, A. (1997). Syntactic Theory and the Structure of English - A Minimalist Approach, Cambridge : C.U.P.

Radford, A. (2009). Analysing English Sentences - A Minimalist Approach, Cambridge : C.U.P. 
Ross, John R. (1972). ‘The category squish : Endstation Hauptwort', In Paul M. Peranteau, Judith N. Levi, Gloria C. Phares (Eds.), Proceedings of the Eighth Regional Meeting of the Chicago Linguistic Society, Chicago Linguistic Society, University of Chicago, Chicago, Illinois, 316-328.

Spang-Hanssen, E. (1963). Les prépositions incolores du français moderne. Copenhague : Forlag.

Svevonius, P. (1996). 'The Optionality of Particle Shift', in Working Papers in Scandinavian Syntax 57, 47-75.

\section{NOTES}

1. Voir également ici même, «La prédication en grammaire générative », §1, note 2 .

2. Le lecteur intéressé pourra se reporter à Khalifa 2004, Ch. 1, § 2.2.2.

3. $C f$. inter al. Jespersen 1924.

4. Rappels que nous réduisons au strict minimum, une bonne partie étant déjà faite dans Khalifa, « La prédication en grammaire générative », ce volume, $\$ 1$.

5. Nous verrons un peu plus loin que cette affirmation doit être sérieusement nuancée, en tout cas pour un traitement unifié des prépositions et des conjonctions.

6. Pour aller vite : la catégorie fonctionnelle I (Inflection) qui accueille les marques de temps, va bien transformer un schéma abstrait d'événement (tout ce qui est dans le VP) en un événement spécifique muni de coordonnées temporelles. De même, la catégorie fonctionnelle D (Determiner) va fournir une délimitation spatiale à un GN qui, sans lui, ne dénoterait que la catégorisation du nom.

7. Remarquons que, à l'époque où est formulé le filtre du cas, on parle encore de noun phrase (NP) et non de determiner phrase (DP), incluant la projection fonctionnelle comme nous l'avons expliqué supra. Ceci ne change strictement rien aux principes d'assignation du cas que nous faisons intervenir au cours de cette étude.

8. ...ou au milieu, peu importe cognitivement.

9. Nous ne pouvons développer cet aspect important mais annexe à notre propos ; voir inter alii Beavers 2011.

10. Ou « coquille de Larson ", du nom du linguiste Richard Larson qui l'introduit dans la théorie dès 1988 ('On the double object construction', Linguistic Inquiry 19: 335-391). Pour aller vite encore, le double VP reflète syntaxiquement l'intuition, au départ sémantique, qu'un événement comme celui dénoté par GIVE comporte en fait deux sous-événements: un événement de déclenchement par un agent, et un événement statif de localisation finale de l'objet transféré par rapport au bénéficiaire.

11. Ceci est bien entendu un raccourci ; la littérature sur ce «mouvement datif » (dative shift) est foisonnante depuis la fin des années 80. L'une des hypothèses avancées (voir inter alii Baker 1997) est que la préposition remonte pour s'incorporer au verbe, en l'occurrence GIVE, ce qui lui permet d'assigner le datif à l'objet second. Il faudrait également expliquer, pour que les choses soient correctement posées, dans quelle position exactement remonte cet objet second, qui ne va pas mécaniquement prendre la place de l'objet direct. Mais nous souhaitions simplement montrer l'interaction entre cas, préposition et mouvement.

12. Nous suivons ici une opinion assez largement partagée: les parties du discours traditionnellement étiquetées "conjonctions" sont à analyser comme des prépositions sélectionnant un CP (ou une proposition, en termes plus traditionnels).

13. Tout au moins corpus d'adultes, car chez les enfants les résultats seraient différents. Dans le C.O.C.A., par exemple, of arrive en $4^{\mathrm{e}}$ position des mots les plus fréquents de l'anglais, derrière $T H E, B E$ et AND. Pour mémoire, les prépositions suivantes, par ordre de fréquence, sont IN, TO, FOR, 
WITH, ON, AT, FROM et BY. Il s'agit là de fréquences globales, nous n'entrons pas dans le détail des registres, l'important est simplement de constater l'extrême fréquence de of en anglais contemporain.

14. Certains linguistes assimilent parfois $T O$ à $O F$ dans cette catégorie de prépositions désémantisées n'ayant pour rôle que d'assigner un cas à leur complément (то assignant, en revanche, le datif). Nous n'en dirons pas davantage, To préposition étant notoirement absent de notre texte.

15. Il serait trop long d'expliquer en détail ce modèle, même s'il est intuitivement assez simple à comprendre : pour donner quelques illustrations, dans le domaine nominal, dog, cat, etc. sont résolument du côté lexical, là où des déterminants comme the, $a$, etc. se situent au pôle opposé. Et entre les deux, on pourra classer des éléments semi-lexicaux comme thing, one, body, etc. (cf. Emonds 1985). Pour le domaine verbal, on trouvera les verbes lexicaux (run, break, etc.) à une extrémité du gradient, et Inflection (c'est à dire les marques de Temps, Aspect, Accord, etc.) à l'autre. Et entre les deux, des éléments comme be, have ou get.

16. Par contraste, ON compte 54956 emplois adverbiaux seulement, contre 578274 prépositionnels et pour IN les chiffres sont encore plus spectaculaires, avec 34411 emplois adverbiaux contre 1691158 prépositionnels.

17. À partir d'ici, nous considérerons, avec bon nombre d'auteurs, que « Prt » couvre adverbes et particules, réputés avoir la même syntaxe.

18. De même plus généralement qu'un verbe ne saurait assigner de cas que sous condition d' adjacence ( $c f$. I want him to leave, mais *I want very much him to leave).

19. Exemples empruntés à Cappelle, op. cit., p. 134, (ex. 153), qui d'ailleurs parle de grey area pour ce qui est de ce genre de construction avec leurs multiples sous-variantes...

\section{RÉSUMÉS}

Dans cette contribution, on propose, à partir du corpus commun, une approche générativiste du domaine prépositionnel, qui dans cette optique regroupe quatre sous-catégories définies par des traits $\{ \pm \mathrm{L}\}$ (lexical), $\{ \pm \mathrm{F}\}$ (fonctionnel), les prépositions semi-lexicales, les prépositions fonctionnelles, les adverbes et les particules. On discute des tests syntaxiques permettant d'opposer ces catégories entre elles et de les regrouper deux par deux, et on montre au passage comment les modules constitutifs de la théorie générativiste, en particulier le filtre du cas et la théorie des rôles-théta, sont mis en jeu pour l'analyse des exemples proposés.

On the basis of the common corpus, this paper discusses the generative approach to the prepositional domain, which is shown to include four subcategories defined by binary features: $\{ \pm \mathrm{L}\}$ (lexical), $\{ \pm \mathrm{F}\}$ (functional), namely semi-lexical prepositions, functional prepositions, adverbs and particles. We discuss some of the syntactic tests traditionally used to distinguish these categories, while showing how central modules in generative theory, especially the case filter and the theta role theory, may contribute to the analysis of the proposed examples. 
INDEX

Keywords : prepositions, adverbs, particles, case filter, theta roles, lexical, functional Mots-clés : prépositions, adverbes, particules, filtre du cas, rôles sémantiques, lexical, fonctionnel

\section{AUTEUR}

\section{JEAN-CHARLES KHALIFA}

Université de Poitiers

Forell-Cerlitep (EA 3816)

jean.charles.khalifa@univ-poitiers.fr 